\title{
Exploring the Impact of Treatment Switching on Overall Survival from the PROfound Study in Homologous Recombination Repair (HRR)-Mutated Metastatic Castration-Resistant Prostate Cancer (mCRPC)
}

\author{
Rachel Evans ${ }^{1}$ - $\cdot$ Neil Hawkins ${ }^{1} \cdot$ Pascale Dequen-O'Byrne ${ }^{1} \cdot$ Charles McCrea $^{2} \cdot$ Dominic Muston $^{3}$. \\ Christopher Gresty ${ }^{2} \cdot$ Sameer R. Ghate ${ }^{3} \cdot$ Lin Fan $^{3} \cdot$ Robert Hettle $^{2} \cdot$ Keith R. Abrams ${ }^{1}$. Johann de Bono ${ }^{4}$. \\ Maha Hussain $^{5}$. Neeraj Agarwal ${ }^{6}$
}

Accepted: 20 August 2021 / Published online: 3 September 2021

(c) The Author(s) 2021

\begin{abstract}
Background In oncology trials, treatment switching from the comparator to the experimental regimen is often allowed but may lead to underestimating overall survival (OS) of an experimental therapy.

Objective This study evaluates the impact of treatment switching from control to olaparib on OS using the final survival data from the PROfound study and compares validated adjustment methods to estimate the magnitude of OS benefit with olaparib. Patients and methods The primary population from PROfound (Cohort A) was included, alongside two populations approved for treatment with olaparib by the European Medicines Agency and US Food and Drug Administration: BRCAm and Cohort $\mathrm{A}+\mathrm{B}$ (excluding the PPP2R2A gene). Five methods were explored to adjust for switching: excluding or censoring patients in the control arm who receive subsequent olaparib, Rank Preserving Structural Failure Time Model (RPSFTM), Inverse Probability of Censoring Weights, and Two-Stage Estimation.

Results The RPSFTM was considered the most appropriate approach for PROfound as the results were robust to sensitivity analysis testing of the common treatment effect assumption. For Cohort A, the final OS hazard ratio reduced from 0.69 (95\% CI 0.5-0.97) to between $0.42(0.18-0.90)$ and $0.52(0.31-1.00)$ for olaparib versus control, depending on the RPSFTM selected. Median OS reduced from 14.7 months to between 11.73 and 12.63 months for control.

Conclusions The magnitude of the statistically significant $(P<0.05)$ survival benefit of olaparib versus control observed in Cohort A of PROfound is likely to be underestimated if adjustment for treatment switching from control to olaparib is not conducted. The RPSFTM was considered the most plausible method, although further development and validation of robust methods to estimate the magnitude of impact of treatment switching is needed.
\end{abstract}

\section{Introduction}

Rachel Evans

rachel.evans@visibleanalytics.co.uk

Visible Analytics, 3 Kings Meadow, Oxford OX2 0DP, UK

2 AstraZeneca, Cambridge, UK

3 Center for Observational and Real-World Evidence, Merck \& Co., Inc., Kenilworth, NJ, USA

4 Institute of Cancer Research and Royal Marsden Hospital, London, UK

5 The Robert H. Lurie Comprehensive Cancer Center, Northwestern University Feinberg School of Medicine, Chicago, IL, USA

6 Huntsman Cancer Institute, University of Utah, Salt Lake City, UT, USA
In recent years, the emergence of new treatments for metastatic castration-resistant prostate cancer (mCRPC) has led to improved survival outcomes [1]. This includes novel molecularly targeted agents, such as olaparib, an oral poly(ADPribose) polymerase inhibitor (PARPi), which has been investigated in the PROfound trial (NCT02987543) [2, 3].

PROfound is a prospective, randomized, open-label, phase III trial that included patients with mCRPC who had one or more of 15 homologous recombination repair (HRR) gene alterations based on prospective tissue testing and who had experienced disease progression while receiving a new hormonal agent. Patients with BRCAl/2 germline mutations [4] have known sensitivity to targeted therapy with PARPi $[5,6]$. 


\section{Key Points}

Olaparib is the first and only PARPi approved for mCRPC based on positive phase III evidence.

Olaparib demonstrated a significant improvement in overall survival compared to control in PROfound.

However, almost $70 \%$ of patients randomized to the control arm switched to olaparib following disease progression.

This study explores validated methods to adjust for control patients switching to olaparib using the final data from PROfound.

All methods explored demonstrate that the observed overall survival results are likely to be underestimated.

Patients were assigned to cohorts depending on their gene alterations: patients with at least one alteration in BRCA1, BRCA2, or ATM were assigned to Cohort A ( $n$ $=245)$; patients with alterations in any 12 other prespecified genes were assigned to the exploratory Cohort B $(n=142)$. Patients were randomized (2:1) to olaparib (300 mg twice daily) or investigator's choice of new hormonal agent (enzalutamide $160 \mathrm{mg}$ once daily or abiraterone $1,000 \mathrm{mg}$ once daily plus prednisone $5 \mathrm{mg}$ twice daily, hereafter called 'control'). Overall survival (OS) was a key secondary endpoint for Cohort $\mathrm{A}$, which was assessed in a hierarchical fashion. The patients randomized to control could switch over to treatment with olaparib upon confirmed radiological disease progression and meeting all eligibility criteria.

Olaparib is the first and only PARPi approved for mCRPC based on positive phase III evidence [2, 3]. Olaparib was approved by the US Food and Drug Administration (FDA) in patients with mCRPC and 14 somatic and/or germline HRR-mutated gene alterations (excluding the PPP2R2A gene) in patients who had experienced disease progression while receiving enzalutamide or abiraterone [7]. Olaparib has also been approved by the European Medicines Agency (EMA) in patients with mCRPC who had experienced disease progression while receiving a new hormonal agent and with BRCAm gene alterations [8].

Results from PROfound reported an OS benefit for patients treated with olaparib compared with control across Cohort A, A+B, and BRCAm populations [2, 3]. For the Cohort A, A+B, and BRCAm populations, OS hazard ratios (HRs) for olaparib compared with control were 0.69 (95\% confidence interval [CI] 0.50-0.97), 0.79 (0.61-1.03) [3], and $0.63(0.42-0.95)$ [8], respectively; the OS results in
Cohort A were statistically significant. The OS endpoint for other populations was not included in statistical testing. There was no observed benefit in Cohort B. However, these results may underestimate the effect of olaparib as around $66-69 \%$ of patients in the control arm switched to olaparib, depending on the population considered.

Treatment switching ('switching') in oncology trials is common [9]. For the PROfound trial it was agreed with the steering committee and regulatory bodies (FDA and EMA) that switching from control to olaparib should be allowed upon radiographic progression (blinded independent central review (BICR)-confirmed up to data cutoff (DCO) 1 and investigator-assessed beyond DCO1) if appropriate for the patient.

For Health Technology Assessment and payer purposes, it is important to understand the incremental benefit of the new treatment compared with standard of care without results being confounded by switching. Various statistical methods exist to adjust OS outcomes for switching, including 'naïve' methods, such as censoring or excluding patients who switch from the analysis to more 'complex' methods, such as Rank Preserving Structural Failure Time Models (RPSFTM), Inverse Probability of Censoring Weights (IPCW), and TwoStage Estimation (TSE). These methods are acknowledged and accepted by Health Technology Assessment agencies and regulatory bodies [10-12]. Identifying the appropriate adjustment method for PROfound is key to estimating the magnitude of OS benefit for patients treated with olaparib had switching to olaparib from control not occurred.

Hussain et al. have partially explored adjusting for switching in PROfound as part of a prespecified sensitivity analysis using the RPSFTM for Cohort A, Cohort B, and Cohort A+B [3]. It has also been explored using the interim OS data [13].

The objective of this study is to evaluate the impact of switching on OS using the final data, comparing published adjustment methods and identifying the most appropriate method. We consider Cohort A and two further populations approved for olaparib treatment by the EMA and US FDA: BRCAm and Cohort A+B (excluding the PPP2R2A gene).

\section{Methods}

\subsection{Patients}

Detailed methodology and eligibility criteria for PROfound have been published elsewhere [2].

In this study, the results for OS adjusted for switching in the control arm to olaparib were primarily explored in Cohort A. Two secondary analyses were also included. One analysis in those with a BRCAm as included in the EMA label [8], and is the largest gene subgroup in the study $(n=160)$, and a predefined subgroup analysis in PROfound. 
An analysis in the FDA-approved population was also included, that is those in Cohort $\mathrm{A}+\mathrm{B}$ (minus PPP2R2A) [7] $(n=372)$. Methodology and selected results pertaining to Cohort $\mathrm{A}+\mathrm{B}$ (minus PPP2R2A) population are included in the Online Supplementary Material (OSM).

Following disease progression, subjects randomized to the control arm were eligible to receive olaparib, given no subsequent anti-cancer therapies were received following discontinuation, and unresolved toxicities from prior therapy were resolved and no greater than Common Terminology Criteria for Adverse Events grade 1 at time of starting olaparib. At the prespecified interim analysis for OS (DCO1, 4 June 2019), patients were eligible to receive subsequent olaparib after BICR-assessed radiographic progression. BICR assessments of progression were stopped at DCO1 following positive primary PFS results [3]. From DCO1 to the time of the final analysis (DCO2, 20 March 2020), patients were eligible to receive subsequent olaparib after investigator-assessed radiographic progression; $67 \%$ and $69 \%$ of patients randomized to the control arm received subsequent olaparib in Cohort $\mathrm{A}$ and the BRCAm subgroup, respectively.

\subsection{Endpoint and Statistical Analysis}

The endpoint for this analysis is OS. Detailed results for the primary endpoint (rPFS) and other secondary endpoints have been previously reported $[2,3]$. OS (defined as time from randomization to death from any cause) was an alpha controlled, key secondary endpoint in Cohort A of PROfound. Kaplan-Meier (KM) curves and Cox HRs are presented for each method and population.

Five switching methods were investigated: "naïve methods' (excluding switchers, censoring switchers) and 'complex methods' (RPSFTM, IPCW, TSE). Methods are summarized in Table 1 and more detail has been provided in the OSM. A priori, there is often no clear best method for adjustment as it depends upon study design, conduct and patient characteristics. The National Institute for Health and Care Excellence (NICE) guidelines, referenced by the EMA, recommends all methods and associated assumptions are explored to select the most appropriate on a study-by-study basis $[10,11,14]$.

Naïve methods do not adjust for patient characteristics. These methods are prone to selection bias if switching is associated with patient characteristics, which breaks the randomization balance. In the PROfound trial, the decision to switch is made by the investigator and patient, and likely based on patient characteristics and preferences.

The RPSFTM approach estimates the causal effect of treatment using a counterfactual framework, where counterfactual survival times are those that would have been observed if switching from control to the experimental arm had not occurred [15]. The counterfactual survival times for control are equivalent to the time spent on control plus the time spent on olaparib multiplied by an 'acceleration factor', which was calculated using different models (see Table 1). The acceleration factor used should be that for which the survival times between the two randomized groups are equal, before treatment with olaparib has been received. The acceleration factor is applied to the survival times for the switchers in the control arm before treatment effect estimates are recalculated for olaparib compared with adjusted control.

The RPSFTM relies on the clinical and biological plausibility of the randomization assumption and common treatment effect assumption (see Table 1). Plots of the counterfactual times between the reference and comparator arms were compared to test the randomization assumption, and to test the common treatment effect assumption a threshold analysis on the treatment effect received by switchers is applied in a sensitivity analysis [16].

The TSE method also uses a counterfactual framework. TSE should only be used to adjust for switching that occurs after a specific disease-related time point, called the 'secondary baseline'. Disease progression is commonly used, as trials, such as PROfound, often stipulate that patients are only permitted to switch treatments once disease progression has occurred. Disease progression would be an unsuitable secondary baseline if patients deviated from the trial protocol, or if the spread of switch times after disease progression is wide and confounding exists between the secondary baseline and point of switch.

The IPCW method extends the censoring at point-ofswitch analysis, by applying weights to patients according to their probability of switching treatments. It aims to remove selection bias introduced by censoring switchers by reweighting non-switchers according to an estimated probability, based on covariables, that they would have switched. The IPCW analysis relies on the "no unmeasured confounders' assumption (see Table 1) and can be prone to error when there is a small sample size with very large (or small) switching proportions.

Detailed methodology of all methods is given in the OSM.

\section{Results}

\subsection{Patient Population}

Baseline characteristics for the olaparib and control arms have been previously reported for Cohort A, Cohort A+B and the BRCAm subgroup [2,17]. Baseline characteristics for the control arm for switchers and non-switchers, for Cohort A and the BRCAm subgroup, are included in Table 2. Characteristics for Cohort $\mathrm{A}+\mathrm{B}$ (minus PPP $2 R 2 A$ ) population are included in the OSM. 
Table 1 Summary of methodology

\begin{tabular}{|c|c|c|}
\hline Method & & Summary description $^{\mathrm{a}}$ \\
\hline Naïve & Excluding switchers & $\begin{array}{l}\text { Patients randomized to the control arm who subsequently received olaparib were identified and removed from } \\
\text { the analysis } \\
\text { Less computationally expensive than the sophisticated methods }\end{array}$ \\
\hline & Censoring switchers & $\begin{array}{l}\text { Patients randomized to the control arm who subsequently received olaparib were censored from the analysis at } \\
\text { point of switching } \\
\text { Less computationally expensive than the sophisticated methods }\end{array}$ \\
\hline Complex & RPSFTM & $\begin{array}{l}\text { Causal effect of treatment is estimated using counterfactual framework, where counterfactual survival times are } \\
\text { those that would have been observed if treatment switching had not occurred } \\
\text { The counterfactual survival times for control are equivalent to the time spent on control plus the time spent } \\
\text { on olaparib multiplied by an 'acceleration factor'. This acceleration factor is the degree to which being on } \\
\text { olaparib increases survival and can be calculated using different models, including log rank, Cox proportional } \\
\text { hazards, and Weibull, all of which were evaluated in this study [24]. } \\
\text { Another consideration when estimating acceleration factors is the application of recensoring, which is where a } \\
\text { trial participant is recensored at the minimum possible censoring time to avoid bias induced by informative } \\
\text { censoring } \\
\text { Hussain et al. [3] have explored adjusting for switching in PROfound as part of a prespecified sensitivity analy- } \\
\text { sis using the RPSFTM (including recensoring; Cox proportional hazards model) } \\
\text { Key assumptions: } \\
\text { Randomization assumption: randomization means that the two groups are comparable and that if they had both } \\
\text { received control treatment then their survival would be the same on average } \\
\text { Common treatment effect assumption: that patients who switch onto olaparib following progression have the } \\
\text { same treatment effect on the accelerated time scale compared with patients originally randomized to olaparib. } \\
\text { Given the majority of patients switch after radiological progression (BICR-assessed for DCO1, BICR- or } \\
\text { investigator-assessed for DCO2), and that patients may have a different capacity to benefit from treatment, this } \\
\text { assumption may not be clinically plausible for PROfound }\end{array}$ \\
\hline & TSE & $\begin{array}{l}\text { Causal effect of treatment is estimated using counterfactual framework, where counterfactual survival times are } \\
\text { those that would have been observed if treatment switching had not occurred. The TSE method should only } \\
\text { be used to adjust for switching that occurs after a specific disease-related time point, which is labelled as the } \\
\text { 'secondary baseline'. The effect of the new treatment on survival can then be estimated by comparing survival } \\
\text { within the control arm from the secondary baseline onwards, between those who switch and do not switch } \\
\text { Key assumptions: } \\
\text { No unmeasured confounders } \\
\text { No time-dependent confounding on treatment switching after disease progression }\end{array}$ \\
\hline & IPCW & $\begin{array}{l}\text { An extension of the censoring at point of switch per-protocol analysis, which applies weights to patients accord- } \\
\text { ing to their probability of switching treatments, modelled using baseline and time dependent variables that } \\
\text { influence the probability of switching and are prognostic of survival. A sensitivity analysis can be performed } \\
\text { using all available variables } \\
\text { The goal is to remove the selection bias introduced by censoring switchers by reweighting non-switchers } \\
\text { according to an estimated probability based on covariables that they would have switched } \\
\text { Individuals in the control arm who have not switched treatments but have similar characteristics to those that } \\
\text { have switched treatments are weighted more highly, to account for their outcomes and also the outcomes of } \\
\text { patients with similar characteristics to them but who switched treatments and were therefore censored from } \\
\text { the dataset } \\
\text { Key assumption: } \\
\text { No unmeasured confounders: all baseline covariates and time-dependent confounders that predict switching } \\
\text { and outcomes are included. This may not hold when there is relatively little prognostic data collected post- } \\
\text { randomization, limiting the scope of time-varying covariables that can be included in an analysis }\end{array}$ \\
\hline
\end{tabular}

$B I C R$ blinded independent central review, DCO data cutoff, IPCW Inverse Probability of Censoring Weights, RPSFTM Rank Preserving Structural Failure Time Model, TSE Two-Stage Estimation

${ }^{a}$ Detailed methodology is provided in the Online Supplementary Material 


\subsection{Outcomes}

\subsubsection{Cohort A}

The OS HR for olaparib compared with control without adjustment for switching in the control arm is $0.69(95 \%$ CI 0.5-0.97) [3]. All HR results for analysis adjusted for switching are provided in Fig. 1.

\subsubsection{Rank Preserving Structural Failure Time Models} (RPSFTMs) The HR for olaparib compared with control ranged between 0.42 (95\% CI 0.18-0.9) and 0.52 (95\% CI 0.31-1.00) depending on the model selected (i.e., log rank, Cox and Weibull; with or without recensoring). Median survival estimates were between 11.73 and 12.63 months for the adjusted control arm, compared with 14.70 months for the unadjusted control. The acceleration factors were broadly similar between models (see OSM). Differences between the estimates including or excluding recensoring may be explained by the shape of the KM curves presented in Fig. 2A, B: when recensoring is not included, the treatment and comparator curves meet, or almost meet, and plateau; whereas for the recensored curves, the treatment and comparator arms do not meet, because the patients were recensored before this point, which changes the shape of the curves.

Results of the RPSFTM were robust to deviations from the common treatment effect assumption. The randomization assumption was considered to hold (see OSM).

3.2.1.2 Two-Stage Estimation (TSE) TSE can be applied when switching occurs immediately after a disease-related time point (secondary baseline) [18]. BICR-confirmed radiological progression was considered; however, it was not found to be an adequate predictor of imminent switching, with only $38 \%$ of control arm switchers switching to olaparib within 30 days of radiological progression. The reasons for delayed switching to olaparib after progression were not collected and likely driven by multiple factors. Furthermore, there was an added complication of identifying a secondary baseline due to switching from control to olaparib being allowed to occur after BICR-assessed progression up to DCO1 and based on investigator-assessed progression from DCO1 to DCO2. Other events were considered, but a reliable secondary baseline could not be identified, and therefore the analysis was not performed.

\subsubsection{Inverse Probability of Censoring Weights} (IPCW) The central HR for olaparib compared to control was estimated to be 0.57 (95\% CI 0.33-0.97). The median OS for adjusted control was 14.01 months and the KM curves are presented in Supplementary Fig. 4 (OSM).
The HR for the sensitivity analysis, which calculates weights using all available variables for olaparib compared to control, was 0.59 (95\% CI 0.35-1.01) (see OSM).

Only 27 (33\%) patients in the Cohort A control arm did not switch to olaparib, and so there may be some bias present in the results.

3.2.1.4 Naïve Methods For the naïve approaches, the HR for OS for olaparib compared with control was 0.39 (95\% CI 0.24-0.65) when switchers were excluded and 0.69 (95\% CI 0.42-1.14) when switchers were censored. For KM curves see the OSM.

\subsubsection{BRCAm Subgroup}

All results are provided in Fig. 3. Findings for this subgroup are consistent with those for Cohort A.

3.2.2.1 RPSFTM For the RPSFTM, the HR for olaparib compared with control ranges between 0.27 (95\% CI 0.19-0.75) and 0.40 (95\% CI 0.27-0.90) depending on model selected. Median survival was between 9.15 and 10.16 months for the adjusted control arm, compared with 14.45 months for the unadjusted control. KM curves are presented in Fig. 2C, D.

3.2.2.2 IPCW and TSE For the IPCW method, the HR for olaparib compared with control was 0.40 (95\% CI $0.21-$ 0.76), and the median OS for adjusted control was 14.01 months. TSE was deemed inappropriate for the same reasons as Cohort A.

3.2.2.3 Naïve Methods For the naïve approaches, the HRs for OS for olaparib compared with control were 0.22 (95\% CI 0.11-0.41) excluding switchers and 0.50 (95\% CI $0.27-$ 0.95 ) when censoring switchers.

Selected results pertaining to the Cohort $\mathrm{A}+\mathrm{B}$ (minus $P P P 2 R 2 A)$ population are included in the OSM.

\section{Discussion}

Overall survival is considered an integral endpoint in cancer studies [19, 20]. In PROfound, a high proportion of patients in the control arm switched to olaparib treatment after progression (67\% in Cohort A after a median follow-up of 21.04 months in the control arm), potentially confounding the observed survival outcomes. All adjustment methods evaluated demonstrate that the survival benefit for olaparib is likely to be greater than that observed in PROfound if switching from control to olaparib is adjusted for. This was consistent across all populations assessed. The most considerable improvement in OS is observed in the BRCAm 


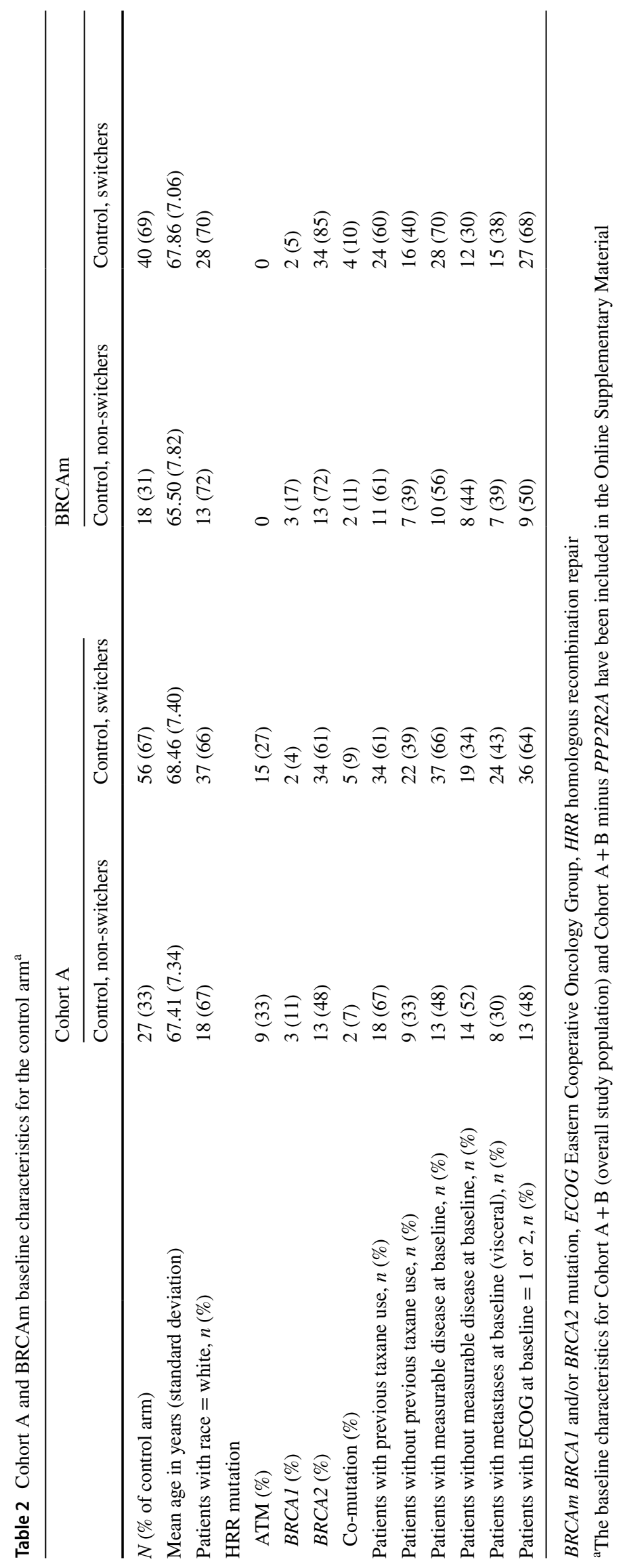


Fig. 1 Overall survival hazard ratios (HRs) for all methods, Cohort A. The dashed line represents unadjusted for switching HR for Cohort A $(n=245)$; the dotted line represents HR $=1.0$; Data used from DCO2: 20 March 2020; all HRs are adjusted for trial stratification factors as in the intention-totreat analysis [2, 3]; see the Online Supplementary Material for the results for Cohort A $+\mathrm{B}$ minus PPP2R2A. DCO data cutoff, IPCW Inverse Probability of Censoring Weights, RPSFTM Rank Preserving Structural Failure Time Model

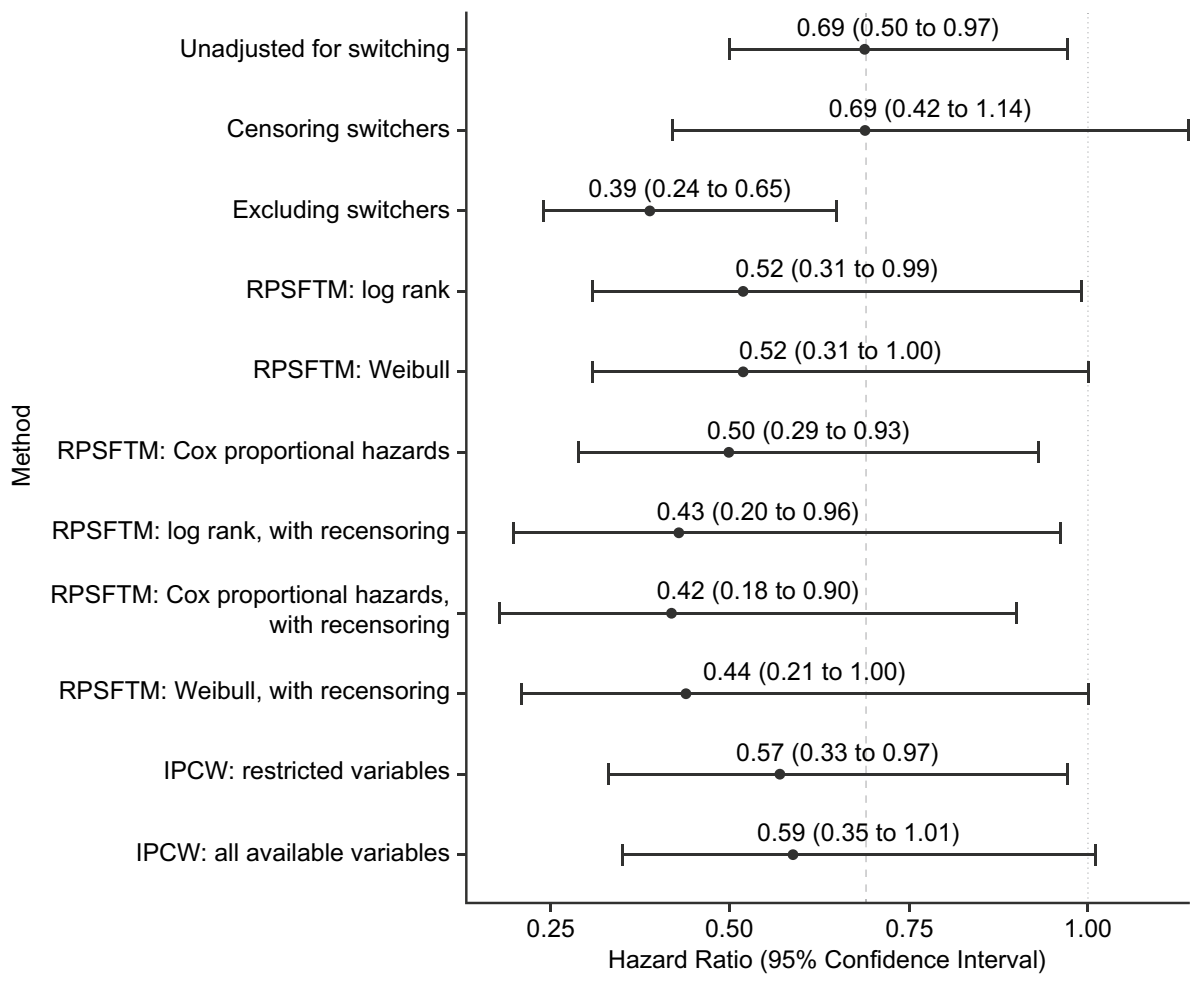

subgroup, which is also the subgroup that derives the greatest survival benefit in the unadjusted analysis compared with other HRR-mutated subgroups [17].

The RPSFTM was considered the most appropriate approach for the PROfound trial. Sensitivity analyses showed the RPSFTM results were robust to deviation from the common treatment effect assumption, and the randomization assumption was considered to hold. When switching in Cohort A was adjusted for using the RPSFTM, the HR was reduced from 0.69 to between 0.42 and 0.52 , with consistency in the direction of the effect across results using different modelling distributions. This aligns with the prespecified analysis published by Hussain et al. [3]. The application of recensoring had the most impact on the results. Recensoring may, however, produce biased results as there are particular features of the treatment effect that appear at longer follow-up times, and it effectively disregards this information [21]. Conversely, not recensoring may also produce bias if extrapolated curves in the long run are not considered clinically plausible.

The other methods considered in this study were found to introduce more bias than the RPSFTM. For the naive methods, the lack of adjustment for patient characteristics made the analysis prone to selection bias. The TSE method was the only method that could be discounted a priori due to the problems encountered with identifying a secondary baseline. The time lag between progression (secondary baseline) and switch day for some patients meant that by the time switched patients started to die there were very few non-switching patients alive. The IPCW method can be biased in situations where there are a larger proportion of switchers [14]. For example, a simulation study has shown high levels of bias can be produced when switching proportions exceeded $85 \%$ [22]. In this analysis, only 27 patients in the control arm did not switch to olaparib in Cohort A, and these patients form the basis of the IPCW control survival estimates. This small sample size increases the amount of uncertainty associated with the result. Furthermore, compelling predictors of switching could not be identified.

Future analysis could consider more sophisticated versions of the complex methods. For example, Latimer et al. (2020) have produced an 'improved' TSE that directly addresses the issue of the time-dependent confounding between disease progression date and switch date in PROfound [23].

Future research could consider the implications of adjusting for switching with small sample sizes. For PROfound, a key limitation applicable to all methods is the sample size of the control arm. This is particularly problematic for the IPCW and TSE, which reduce sample size in the analysis, leading to greater uncertainty in the results. This issue causes further problems for subgroup analysis. The BRCAm patients represent a subgroup of more homogenous patients who have been identified as deriving a clinically meaningful benefit from olaparib treatment [8]. As this is a distinct subgroup, standard methodology dictates that they be selected at the start of the analysis, as 
Fig. 2 Kaplan-Meier curves for Cohort A and BRCAm, adjusted for treatment switching using RPSFTM. A Cohort A, RPSFTM using Cox model without recensoring; B Cohort A, RPSFTM using Cox model with recensoring; C BRCAm, RPSFTM using Cox model without recensoring; D BRCAm, RPSFTM using Cox model with recensoring. bid twice daily, BRCAm BRCA1 and/or BRCA2 mutation, RPSFTM Rank Preserving Structural Failure Time Model

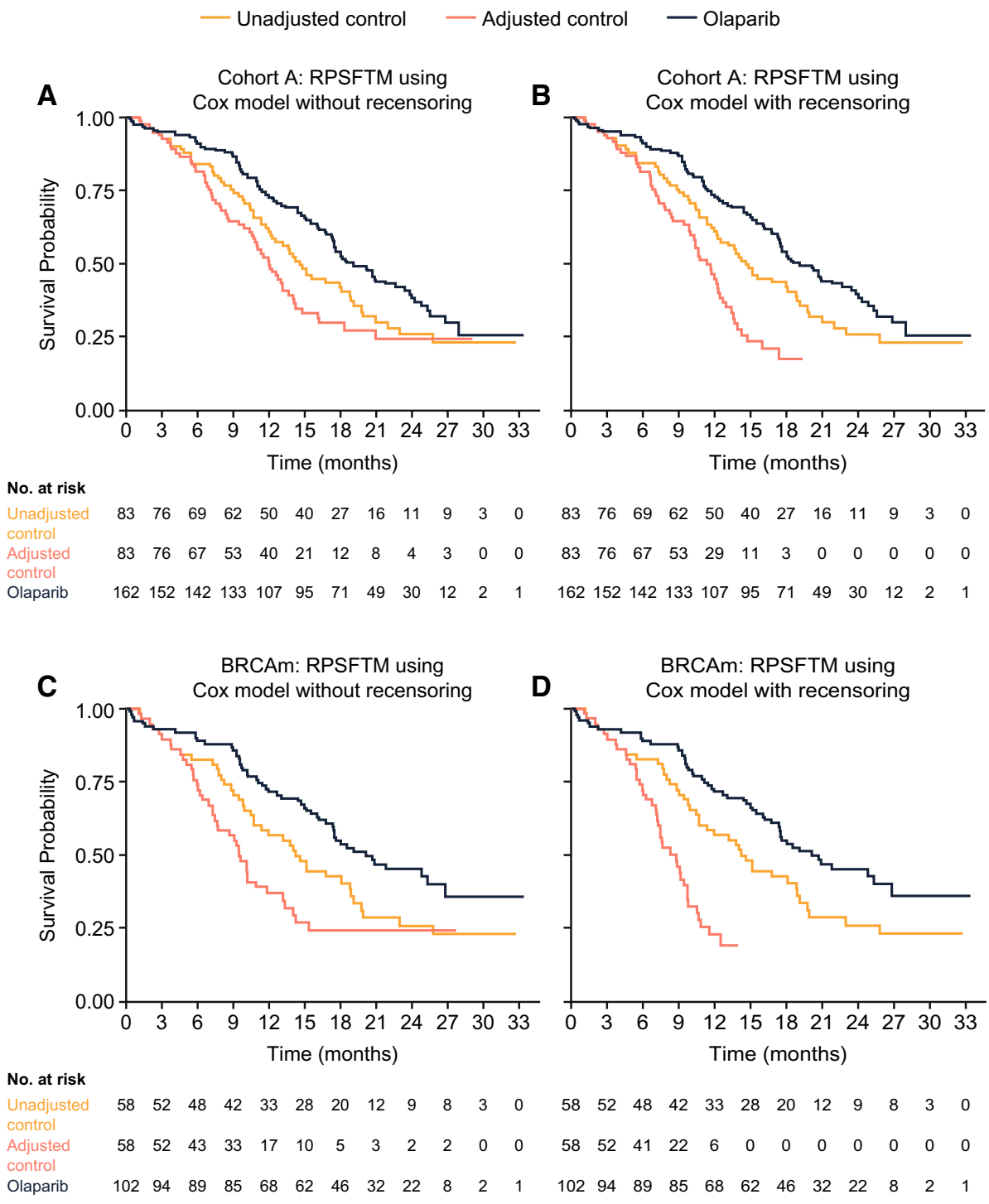

has been presented here. However, this reduces the sample size to 160 patients (58 in control). An alternative method involves calculating counterfactual survival times on all patients and then performing the survival analysis on the BRCAm patients. This approach borrows information from the total population, which may lead to more certain estimates. However, this would mean the weights and counterfactual times are influenced by a patient population that is more heterogeneous than the population of interest and judgement is required as to whether the assumption of exchangeability is valid. Further work exploring hierarchical approaches is warranted.

\section{Conclusion}

These results demonstrate that the observed survival benefit of olaparib versus control in the intention-to-treat population of PROfound is likely to be underestimated. The RPSFTM is a plausible method to adjust for this switching in the PROfound study, but further development and validation of robust and flexible methods to estimate the magnitude of impact of switching are needed. In PROfound, the observed OS and switching analyses demonstrate a survival benefit for olaparib in mCRPC compared with investigator's choice of new hormonal agent. 
Fig. 3 Overall survival hazard ratios (HRs) for all methods, BRCAm. The dashed line represents unadjusted for switching HR for BRCAm $(n=160)$; the dotted line represents $\mathrm{HR}=1.0$; Data used from DCO2: March 20, 2020; all HRs are adjusted for trial stratification factors as in the intention-to-treat analysis [2, 3]; see the Online Supplementary Material for the results for Cohort $\mathrm{A}+\mathrm{B}$ minus PPP2R2A. BRCAm BRCA1 and/ or $B R C A 2$ mutation, $D C O$ data cutoff, IPCW Inverse Probability of Censoring Weights, RPSFTM Rank Preserving Structural Failure Time Model

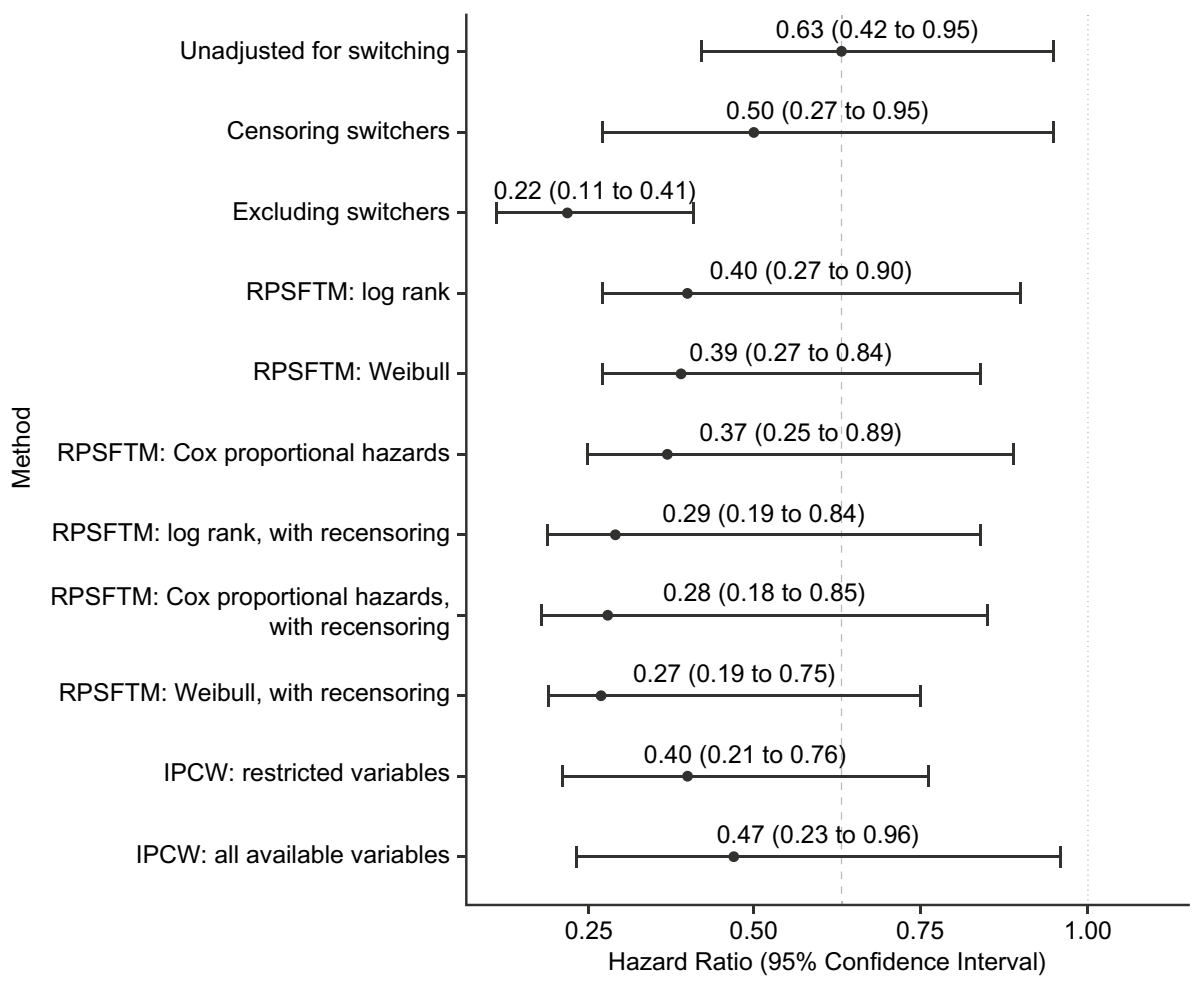

Supplementary Information The online version contains supplementary material available at https://doi.org/10.1007/s11523-021-00837-y.

Acknowledgements The results of this analysis have been presented in part as follows: De Bono J, Mateo J, Fizazi K et al. Olaparib for metastatic castration-resistant prostate cancer. N Engl J Med 382(22):2091-2102, 2020. https://doi.org/10.1056/NEJMoa1911440. Hussain M, Mateo J, Fizazi K et al. Survival with olaparib in metastatic castration-resistant prostate cancer. N Engl J Med 383(24):2345-2357, 2020. https://doi.org/10.1056/NEJMoa1911440. Agarwal N, Evans R, Abrams $\mathrm{K}$ et al. 622P Exploring the impact of treatment switching on the interim overall survival (OS) results of the PROfound study. Ann Oncol 31:S514, 2014. https://doi.org/10.1016/j.annonc.2020.08.881.

\section{Declarations}

Funding This study was funded by AstraZeneca and is part of an alliance between AstraZeneca and Merck Sharp \& Dohme Corp., a subsidiary of Merck \& Co., Inc., Kenilworth, NJ, USA.

Conflict of interest Rachel Evans-Advisory/consultancy: AstraZeneca. Employment: Visible Analytics. Neil Hawkins-Advisory/consultancy: AstraZeneca. Employment: Health Economics and Health Technology Assessment, University of Glasgow, Glasgow, UK. Employee of Visible Analytics. Pascale Dequen-O'Byrne-Advisory/ consultancy: AstraZeneca. Employment: Visible Analytics. Charles McCrea-Employment and stock ownership: AstraZeneca. Dominic Muston-Employment: Merck. Christopher Gresty-Employment and stock ownership: AstraZeneca. Sameer Ghate-Employment: Merck. Lin Fan-Employment: Merck. Robert Hettle-Employment and stock ownership: AstraZeneca. Keith Abrams-Advisory/consultancy: AstraZeneca. Affiliated (as an employee until 31 December 2020) to: Department of Health Sciences, University of Leicester, Leicester, UK. Partially supported as a UK National Institute for Health Research
(NIHR) Senior Investigator Emeritus (NF-SI-0512-10159). The views expressed are those of the author and not necessarily those of NHS, NHS or Department of Health \& Social Care. Employee of Visible Analytics. Johann de Bono-Advisory boards and received fees from many companies, including: Astra Zeneca, Astellas, Bayer, Bioxcel Therapeutics, Boehringer Ingelheim, Cellcentric, Daiichi, Eisai, Genentech/Roche, Genmab, GSK, Janssen, Merck Serono, Merck Sharp \& Dohme, Menarini/Silicon Biosystems, Orion, Pfizer, Qiagen, Sanofi Aventis, Sierra Oncology, Taiho, Vertex Pharmaceuticals. Employment: The ICR, which have received funding or other support for his research work from AZ, Astellas, Bayer, Cellcentric, Daiichi, Genentech, Genmab, GSK, Janssen, Merck Serono, MSD, Menarini/Silicon Biosystems, Orion, Sanofi Aventis, Sierra Oncology, Taiho, Pfizer, Vertex, and which has a commercial interest in abiraterone, PARP inhibition in DNA repair defective cancers and PI3K/AKT pathway inhibitors (no personal income). Patent: Named as an inventor, with no financial interest, for patent $8,822,438$. He has been the CI/PI of many industry sponsored clinical trials. JDB is a National Institute for Health Research (NIHR) Senior Investigator. The views expressed in this article are those of the author(s) and not necessarily those of the NHS, the NIHR, or the Department of Health. Maha Hussain-Grant support (paid to Northwestern University): AstraZeneca, Prostate Cancer Clinical Trials Consortium. Advisory board fees, travel support and grant support (paid to Northwestern University): Bayer. Advisory board fees, travel support and grant support (paid to University of Michigan): Pfizer. Advisory board fees, lecture fees, travel support and grant support (paid to Northwestern University): Genentech. Travel support and honoraria (lecture): Astellas. Honoraria (lecture): Physicians' Education Resource ${ }^{\circledR}$, Sanofi/Genzyme, Phillips Gilmore Oncology, MLI Peer Review. Honoraria (educational material): Projects in Knowledge. Honoraria (educational review): Research to Practice. Neeraj Agarwal-Advisory board fees: Astellas Pharma, AstraZeneca, Bayer, Bristol-Myers Squibb, Clovis Oncology, Eisai, Eli Lilly, EMD Serono, Exelixis, Foundation Medicine, Genentech, Janssen Biotech, Merck, Nektar Therapeutics, Novartis, Pfizer, Pharmacyclics (an AbbVie company), Seattle Genetics. 
Ethics approval This trial was performed in accordance with the principles of the Declaration of Helsinki, Good Clinical Practice guidelines, and the AstraZeneca and Merck policies on bioethics.

Consent to participate All patients provided written informed consent to participate.

Consent for publication All authors have reviewed the final submitted document and agreed to publication.

Availability of data and material Data underlying the findings described in this article may be obtained in accordance with AstraZeneca's data-sharing policy described at https://astrazenecagrouptria ls.pharmacm.com/ST/Submission/Disclosure.

\section{Code availability Not applicable.}

Author contributions Conception and design: Rachel Evans; Neil Hawkins; Pascale Dequen-O’Byrne; Charles McCrea; Robert Hettle; Keith R. Abrams. Acquisition of data: Christopher Gresty. Analysis and interpretation of data: Rachel Evans; Neil Hawkins; Pascale Dequen-O'Byrne; Charles McCrea; Dominic Muston; Christopher Gresty; Sameer R. Ghate; Lin Fan; Robert Hettle; Keith R. Abrams; Johann de Bono; Maha Hussain; Neeraj Agarwal. Drafting of the manuscript: Rachel Evans; Neil Hawkins; Pascale Dequen-O'Byrne; Charles McCrea; Dominic Muston; Christopher Gresty; Sameer R. Ghate; Lin Fan; Robert Hettle; Keith R. Abrams; Johann de Bono; Maha Hussain; Neeraj Agarwal. Critical revision of the manuscript for important intellectual content: Rachel Evans; Neil Hawkins; Pascale Dequen-O'Byrne; Charles McCrea; Dominic Muston; Christopher Gresty; Sameer R. Ghate; Lin Fan; Robert Hettle; Keith R. Abrams; Johann de Bono; Maha Hussain; Neeraj Agarwal. Statistical analysis: Rachel Evans; Neil Hawkins; Pascale Dequen-O'Byrne; Charles McCrea; Christopher Gresty; Robert Hettle; Keith R. Abrams. Obtaining funding: Charles McCrea; Dominic Muston; Sameer R. Ghate; Lin Fan; Robert Hettle. Administrative, technical, or material support: Rachel Evans; Neil Hawkins; Pascale Dequen-O'Byrne; Charles McCrea; Dominic Muston; Christopher Gresty; Sameer R. Ghate; Lin Fan; Robert Hettle; Keith R. Abrams; Johann de Bono; Maha Hussain; Neeraj Agarwal. Supervision: Charles McCrea.

Open Access This article is licensed under a Creative Commons Attribution-NonCommercial 4.0 International License, which permits any non-commercial use, sharing, adaptation, distribution and reproduction in any medium or format, as long as you give appropriate credit to the original author(s) and the source, provide a link to the Creative Commons licence, and indicate if changes were made. The images or other third party material in this article are included in the article's Creative Commons licence, unless indicated otherwise in a credit line to the material. If material is not included in the article's Creative Commons licence and your intended use is not permitted by statutory regulation or exceeds the permitted use, you will need to obtain permission directly from the copyright holder. To view a copy of this licence, visit http://creativecommons.org/licenses/by-nc/4.0/.

\section{References}

1. Nuhn P, De Bono JS, Fizazi K, Freedland SJ, Grilli M, Kantoff $\mathrm{PW}$, et al. Update on systemic prostate cancer therapies: management of metastatic castration-resistant prostate cancer in the era of precision oncology. Eur Urol. 2019;75(1):88-99. https://doi. org/10.1016/j.eururo.2018.03.028.
2. de Bono J, Mateo J, Fizazi K, Saad F, Shore N, Sandhu S, et al. Olaparib for metastatic castration-resistant prostate cancer. N Engl J Med. 2020;382(22):2091-102. https://doi.org/10.1056/NEJMo a1911440.

3. Hussain M, Mateo J, Fizazi K, Saad F, Shore N, Sandhu S, et al. Survival with olaparib in metastatic castration-resistant prostate cancer. N Engl J Med. 2020;383(24):2345-57. https://doi.org/10. 1056/NEJMoa2022485.

4. Lord CJ, Ashworth A. PARP inhibitors: synthetic lethality in the clinic. Science. 2017;355(6330):1152-8. https://doi.org/10.1126/ science.aam7344.

5. Mateo J, Carreira S, Sandhu S, Miranda S, Mossop H, PerezLopez R, et al. DNA-repair defects and olaparib in metastatic prostate cancer. N Engl J Med. 2015;373(18):1697-708. https:// doi.org/10.1056/NEJMoa1506859.

6. Mateo J, Porta N, Bianchini D, McGovern U, Elliott T, Jones $\mathrm{R}$, et al. Olaparib in patients with metastatic castration-resistant prostate cancer with DNA repair gene aberrations (TOPARP-B): a multicentre, open-label, randomised, phase 2 trial. Lancet Oncol. 2020;21(1):162-74. https://doi.org/10.1016/S1470-2045(19) 30684-9.

7. US Food and Drug Administration (FDA). Highlights of prescribing information: LYNPARZA ${ }^{\circledR}$ (olaparib) tablets, for oral use. 2020. https://www.accessdata.fda.gov/drugsatfda_docs/label/ 2020/208558s014lbl.pdf. Accessed 23 Mar 2021.

8. European Medicines Agency (EMA). Lynparza. 2018. https:// www.ema.europa.eu/en/medicines/human/EPAR/lynparza. Accessed 23 Mar 2021

9. Sullivan TR, Latimer NR, Gray J, Sorich MJ, Salter AB, Karnon J. Adjusting for treatment switching in oncology trials: a systematic review and recommendations for reporting. Value Health. 2020;23(3):388-96. https://doi.org/10.1016/j.jval.2019.10.015.

10. Latimer N, Abrams K. NICE DSU Technical Support Document 16: adjusting survival time estimates in the presence of treatment switching. NICE Decision Support Unit, ScHARR; 2014. http:// www.nicedsu.org.uk.

11. European Medicines Agency (EMA). Adjustment for cross-over in estimating effects oncology trials. 2019. https://www.ema.europa. eu/en/adjustment-cross-over-estimating-effects-oncology-trials. Accessed 23 Mar 2021.

12. Latimer NR. Treatment switching in oncology trials and the acceptability of adjustment methods. Expert Rev Pharmacoecon Outcomes Res. 2015;15(4):561-4. https://doi.org/10.1586/14737 167.2015.1037835.

13. Agarwal N, Evans R, Abrams K, Dequen-O'Byrne P, McCrea C, Muston D, et al. Exploring the impact of treatment switching on the interim overall survival (OS) results of the PROfound study. Ann Oncol. 2020;31:S514. https://doi.org/10.1016/j.annonc.2020. 08.881 .

14. Latimer NR, Abrams KR, Lambert PC, Morden JP, Crowther MJ. Assessing methods for dealing with treatment switching in clinical trials: a follow-up simulation study. Stat Methods Med Res. 2018;27(3):765-84. https://doi.org/10.1177/0962280216642264.

15. Robins JA, Tsiatis AA. Correcting for non-compliance in randomized trials using rank preserving structural failure time models. Commun Stat Theory Methods. 1991;20(8):2609-31. https://doi. org/10.1080/03610929108830654.

16. Latimer NR. Treatment switching in clinical trials: Issues for committee members to be aware of. Part 2: What are the adjustment methods. 2020. http://nicedsu.org.uk/training/online-training/. Accessed 23 Mar 2021.

17. De Bono JS, Matsubara N, Penel N, Mehra N, Kolinsky MP, Bompas E, et al. Exploratory gene-by-gene analysis of olaparib in patients (pts) with metastatic castration-resistant prostate cancer (mCRPC): PROfound. J Clin Oncol. 2020;39:126. https://doi.org/ 10.1200/JCO.2021.39.6_suppl.126. 
18. Latimer NR, Abrams KR, Lambert PC, Crowther MJ, Wailoo AJ, Morden JP, et al. Adjusting survival time estimates to account for treatment switching in randomized controlled trials—an economic evaluation context: methods, limitations, and recommendations. Med Decis Mak. 2014;34(3):387-402. https://doi.org/10.1177/ $0272989 X 13520192$.

19. European Medicines Agency (EMA). Guideline on the clinical evaluation of anticancer medicinal products. EMA/CHMP/205/95 Rev.6 [p24, p44]. 2019. https://www.ema.europa.eu/en/docum ents/scientific-guideline/draft-guideline-evaluation-anticancermedicinal-products-man-revision-6_en.pdf. Accessed 10 Aug 2021.

20. US Food and Drug Administration (FDA). Clinical trial endpoints for the approval of cancer drugs and biologics: Guidance for industry [p9]. 2018. https://www.fda.gov/media/71195/downl oad. Accessed 10 Aug 2021.

21. Latimer NR, White IR, Abrams KR, Siebert U. Causal inference for long-term survival in randomised trials with treatment switching: should re-censoring be applied when estimating counterfactual survival times? Stat Methods Med Res. 2019;28(8):2475-93. https://doi.org/10.1177/0962280218780856.

22. Latimer NR, Abrams KR, Lambert PC, Crowther MJ, Wailoo AJ, Morden JP, et al. Adjusting for treatment switching in randomised controlled trials - a simulation study and a simplified two-stage method. Stat Methods Med Res. 2017;26(2):724-51. https://doi. org/10.1177/0962280214557578.

23. Latimer NR, White IR, Tilling K, Siebert U. Improved two-stage estimation to adjust for treatment switching in randomised trials: g-estimation to address time-dependent confounding. Stat Methods Med Res. 2020;29(10):2900-18. https://doi.org/10.1177/ 0962280220912524.

24. Allison A, White IR, Bond S. rpsftm: an R package for rank preserving structural failure time models. R J. 2017;9(2):342-53. 